\title{
Enhanced Mosquito Surveillance for Aedes spp. in Santa Cruz County, Arizona
}

\author{
Mariana G. Casal ${ }^{\star 1,2}$, Jose Arriola ${ }^{3}$, Steven Erly ${ }^{1,2,4}$, Nicolette Dent ${ }^{1,5}$, Shelly Jacobs ${ }^{3}$, \\ Kacey Ernst ${ }^{4}$, Kathleen Walker ${ }^{4}$ and Mary Hayden ${ }^{6}$
}

'Office of Border Health, Arizona Department of Health Services, Tucson, AZ, USA; ${ }^{2}$ Binational Border Infectious Disease Surveillance Program, Tucson, AZ, USA; ${ }^{3}$ Santa Cruz County Health Services, Nogales, AZ, USA; ${ }^{4}$ University of Arizona, Tucson, AZ, USA; ${ }^{5}$ Centers for Disease Control and Prevention, Office for State, Local, Territorial, and Tribal Support, Atlanta, GA, USA; ${ }^{6}$ National

Center for Atmospheric Research, Fort Collins, CO, USA

\section{Objective}

The objective of this work is to develop an efficient communitybased strategy to enhance mosquito surveillance for Aedes spp., vector for chikungunya and dengue viruses, in Santa Cruz County on the U.S.-Mexico border. We aim to determine vector presence, distribution, and seasonality by using ovitraps maintained by community members.

\section{Introduction}

Since 2003 some Arizona counties have followed mosquito surveillance protocols to trap the West Nile Virus vector, Culex spp., using $\mathrm{CO}_{2}$ traps. Despite low sensitivity of these traps to detect Aedes spp., one out of seven $\mathrm{CO}_{2}$ traps deployed in Santa Cruz County detected Aedes aegypti in 2014. Enhancing surveillance for Aedes spp. in this region is critical, given that local transmission of dengue has occured across the border in Nogales, Sonora. Limited resources in Santa Cruz County have previously inhibited efforts to enhance mosquito surveillance ${ }^{1}$. To broaden the reach of county surveillance, we implemented a community participatory project by engaging residents to conduct ovitrapping, a non-technical trap that attracts Aedes spp. ${ }^{2}$

\section{Methods}

Multiple strategies were employed to recruit community members to conduct the ovitrapping. Key groups approached included high school science clubs, International Baccalaureate programs, and senior citizen groups. During the sessions, program staff reviewed the project and the importance of enhancing Aedes spp. surveillance. Volunteers were instructed on the ovitrap use including how to make a hay infusion, put together the ovitrap, identify eggs, and store and transport specimens for identification. Protocols for ovitrapping were developed jointly by the BIDS program, the University of Arizona, and National Center for Atmospheric Research. Recruitment of participants began in March 2015. Traps were placed as individuals were recruited and data was collected on a weekly basis. Tracking of the frequency of reporting from the community participants was conducted. As samples were sent in, the county reported positive traps to the state and eggs were reared and identified to species. All trap locations were mapped and the presence of Aedes spp. was noted for each collection period

\section{Results}

As of August 3, 2015 a total of 43 trapping sites were set and maintained by community members. These 43 sites supplemented 11 county-maintained sites, seven of which were housed at fire stations. Participants included community members from senior citizen groups $(n=24)$, local high schools $(n=12)$, and other local residents $(n=7)$. The geographic trap distribution included 17 sites in the Nogales area closest to the border, two in the central Patagonia area, and 24 in the Tubac area approximately 25 miles north of the border. Traps were kept in participants' backyards. Of the 43 community participants,
$72 \%(n=31)$ collected data systematically and according to the protocol. Participation rates varied among community members. While senior citizen groups had regular participation rates, a barrier to retaining high school participation was the overlap of summer vacation with the surveillance season.

\section{Conclusions}

By recruiting community members to maintain ovitraps, we tripled the county's surveillance capacity for Aedes spp. Citizen science projects are challenging, but strong protocols and an engaging educational component can harness community labor and gather baseline vector data within a county. Data acquired through this project will allow epidemiologists to anticipate and prevent possible outbreaks at the border.

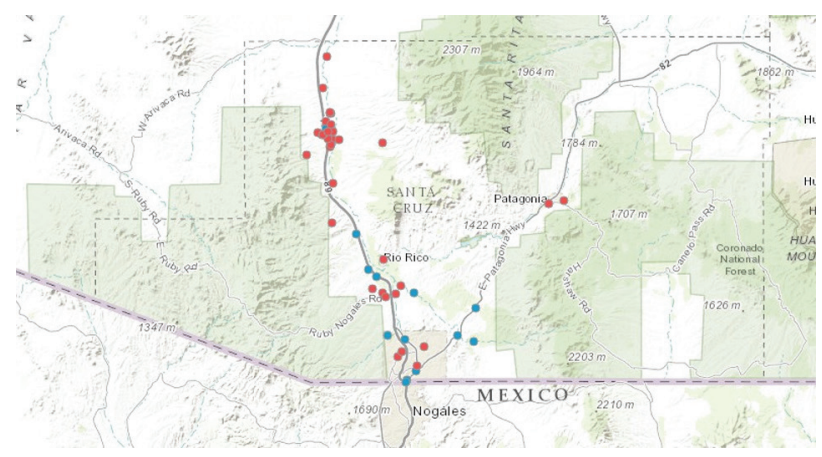

Ovitrap distribution in Santa Cruz County

\section{Keywords}

aedes spp. surveillance; ovitraps; citizen science; mosquito

\section{References}

1. Kampen H, Medlock JM, Vaux AG, et al. Approaches to passive mosquito surveillance in the EU. ;(9). DOI: 10.1186/s13071-0140604-5.

2. Polson KA, Curtis C, Seng CM, et al. The use of ovitraps baited with hay infusion as a surveillance tool for Aedes aegypti mosquitoes in Cambodia. Dengue Bulletin 2002; 26:178-184.

\section{*Mariana G. Casal}

E-mail: mariana.casal@azdhs.gov 